\title{
Inteligência e Criatividade na Maturidade e Velhice
}

\author{
Intelligence and Creativity in Middle and Old Age
}

\author{
Adriana Aparecida Ferreira de Souza, ${ }^{*, b}$ \& Solange Muglia Wechsler ${ }^{b}$ \\ ${ }^{a}$ Universidade de Mogi das Cruzes, Mogi das Cruzes, São Paulo, Brasil \\ \& ${ }^{b}$ Pontifícia Universidade Católica de Campinas, Campinas, São Paulo, Brasil
}

\begin{abstract}
Resumo
Este estudo se propôs a comparar as habilidades intelectuais e criativas entre pessoas na meia idade e idosos. Participaram 81 pessoas $(61,73 \%$ de 45 a 59 anos e $38,27 \%$ acima de 60 anos). Para a coleta de dados foram utilizados a Bateria de Habilidades Intelectuais de Adultos e os testes Pensando Criativamente com Palavras e com Figuras, que foram aplicados individualmente. Os resultados demonstraram que os testes verbais, de processamento visual e criatividade foram influenciados significativamente pelo nível educacional. Os testes de memória sofreram influência significativa de escolaridade, com quedas na faixa etária maior. Inteligência demonstrou estar correlacionada significativamente com criatividade. Conclui-se que a escolaridade é fator significante no desempenho cognitivo e criativo tanto na meia idade quanto na velhice.

Palavras-chave: Envelhecimento, inteligência, habilidades, criatividade, avaliação psicológica.

Abstract

This study aimed to compare intellectual and creative skills of middle-aged and elderly individuals. Eighty one people $(61.73 \%$ from 45 to 59 years old and $38.27 \%$ over 60$)$ participated in the survey. The tests "Intellectual Abilities for Adults" and "Thinking Creatively with Words and Pictures" were used for data collection and were individually administered. The results demonstrated that verbal, visual processing and creativity tests were significantly influenced by the educational level. Memory tests were also under significant influence of schooling and showed greater decrease at older ages. Intelligence and creativity were significantly correlated. In conclusion, schooling is a significant factor affecting cognitive and creative performance in the middle and old age stages.

Keywords: Aging, intelligence, abilities, creativity, psychological assessment.
\end{abstract}

Investigações teóricas e empíricas relacionadas ao conceito de inteligência e da criatividade têm ocupado uma posição de destaque na História da Psicologia. O exame da literatura mostra que os modelos sobre estes construtos vêm evoluindo em um processo cumulativo e integrativo, e que os testes de inteligência estão cada vez mais sofisticados (Oliveira-Castro \& Oliveira-Castro, 2001; Primi, 2002). Entre os modelos mais clássicos para explicar a inteligência está o de Spearman (1927) que defendia que toda atividade intelectual se exprime em um fator geral (Fator G) e o de Thurnstone (1938), que propôs uma mente humana constituída por diferentes e independentes aptidões, a saber: compreensão verbal, fluência verbal, aptidão numérica, velocidade perceptiva, aptidão espacial, memória e raciocínio (Aptidões Primárias).

*Endereço para correspondência: Pontifícia Universidade Católica de Campinas, Curso Pós-Graduação Psicologia, Av. John Boyd Dunlop, s/n, Jardim Ipaussurama, Campinas, São Paulo, Brasil 13104-072.E-mail: adr_ferreira@ hotmail.com

Agradecimentos: A Coordenação de Aperfeiçoamento de Pessoal de Nível Superior (CAPES) pela bolsa de doutorado e aos Grupos de Terceira Idade participantes do estudo.
O trabalho de Cattell $(1941,1971)$ pretendia delimitar fatorialmente a estrutura da inteligência, e resultou em um modelo integrado chamado Gf-Gc (inteligência fluida e inteligência cristializada). Horn (1991), um dos discípulos de Cattell, aprimorou o modelo Gf-Gc. Carroll (1993), por sua vez, utilizou a análise fatorial para estudar 60 anos na literatura científica sobre inteligência. $\mathrm{O}$ resultado foi a teoria dos Três Extratos, que distingue entre as aptidões concretas, amplas e gerais. No terceiro extrato se situa o Fator G, no segundo as aptidões amplas e no primeiro as aptidões específicas (Colom \& Flores-Mendonza, 2001; Primi, 2002).

McGrew e Flanagan (1998) foram os responsáveis pela integração das teorias Gf-Gc (Cattell, Horn) e dos três extratos (Carroll), considerando que os modelos de Carroll e Horn-Cattell possuem grandes semelhanças entre si, já que ambos consideram a existência de capacidades gerais relacionadas. Denominaram a nova teoria de CHC (Cattell-Horn-Carroll) que consiste em uma visão hierárquica multidimensional das habilidades cognitivas. A teoria $\mathrm{CHC}$ estabelece dez fatores do funcionamento cognitivo: Inteligência fluida (Gf), Conhecimento quantitativo (Gq), Inteligência cristalizada (Gc), Leitura e escrita 
(Grw), Memória de curto prazo (Gsm), Processamento visual (Gv), Processamento auditivo (Ga), Habilidade de armazenamento e recuperação da memória de longo prazo (Glr), Velocidade de processamento (Gs) e Rapidez de decisão (Gt; Muñoz-Sandoval \& Woodcock, 2005; Primi, 2002). Nesse sentido, Woodcock decidiu revisar suas duas baterias e adequá-las ao modelo $\mathrm{CHC}$, o que resultou em uma nova bateria, denominada Woodcock-Johnson III / WJ III (Woodcock, McGrew, \& Mather, 2001). Atualmente a Bateria WJ III é considerada pelos especialistas internacionais como a mais completa medida das habilidades cognitivas (Muñoz-Sandoval \& Woodcock, 2005; D. Wechsler, 1949/2003).

Poucos estudos foram encontrados, entretanto, sobre o desenvolvimento inteligência - habilidades cognitivas na faixa etária idosa no Brasil. Considerando-se as teorias de inteligência utilizadas para avaliações psicométricas, pode-se afirmar a provável tendência da Inteligência Cristalizada evoluir com o aumento da idade, ao contrário da Fluida que parece declinar após a idade de 21 anos, devido à degeneração das estruturas fisiológicas (Brody, 2000; Sattler, 2001). Há indícios de que a inteligência fluida tem aumentado significativamente nos dois últimos séculos. Este fato foi comprovado por Flynn (1984) ao comparar os resultados nos testes de QI entre amostras norte-americanas coletados entre 1932 a 1978 , sendo posteriormente comprovados na década de 1980-1990 (Flynn, 1998; Kaufman \& Kaufman, 1983) e no início do século XVI (Flynn, 2007). Possíveis razões para tais aumentos têm sido atribuídas as melhores condição de saúde e educação na população, e que por sua vez demonstram a necessidade dos testes possuírem normas atualizadas para avaliar as habilidades intelectuais entre gerações e diferentes culturas (Kaufman, 2009). Segundo Johnson, Gow, Corley, Starr e Deary (2010) a capacidade mental tende a ser relativamente estável ao longo da vida. Na velhice a função cognitiva reflete amplamente o nível educacional, classe social e circunstâncias ambientais.

Angelini, Alves, Custódio, Duarte e Duarte (1999) encontraram evidência de que a inteligência fluida aumenta de uma geração para outra através do teste Raven. Ribeiro, Oliveira, Cupertino, Néri e Yassuda (2010) avaliaram o desempenho de idosos na Bateria Cognitiva CERAD, comparando a variáveis sócio-demográficas e saúde percebida e também encontraram correlação significante entre saúde percebida e todas as habilidades avaliadas (exceto reconhecimento) com escolaridade, o que também reforça os achados do presente estudo. Por outro lado, há carência de estudos utilizando o modelo mais atual da teoria $\mathrm{CHC}$, que abrange mas habilidades intelectuais do que os testes tradicionais de QI.

A avaliação da criatividade também sofreu avanços nos últimos anos. De acordo com Alencar e Fleith (2004) muitas das ideias preconcebidas deixaram de existir, houve um crescimento e amadurecimento das reflexões; o que era considerado inspiração e dom especial para um grupo de indivíduos privilegiados se transformou em uma nova ideia, isto é, que todo ser humano apresenta certo grau de criatividade, que pode ser treinado e aprimorado. Atualmente a definição de criatividade converge para a característica multidimensional (Nakano, 2003; S. M. Wechsler, 2008).

De acordo com Godoy (1996), a criatividade se manifesta em todas as áreas da atividade humana, entre elas as relações interpessoais, o desempenho no trabalho, a participação social, a busca espiritual e a educação. Dessa forma, faz-se necessário identificar o processo de utilização da criatividade entre as pessoas na meia-idade e velhice. O termo late-life bloomer tem sido utilizado para se referir a pessoas que apenas na velhice tendem a revelar talentos criativos e intelectuais. É uma metáfora a plantas que florescem na primavera, verão e no inverno. Algumas árvores americanas não florescem antes da primeira geada. Por analogia, algumas pessoas mostram seus frutos apenas quando seus cabelos estão grisalhos (Birren, 2009). Relatos sobre o desempenho criativo na meia-idade e velhice são encontrados na literatura internacional, bem como sua relação com saúde mental e satisfação com a vida (Aadlandsvik, 2007; Foster, 1992; Gori, Pientini, \& Vespa, 2001; Reisenwitz, Iyer, Kuhlmeier, \& Eastman, 2007; Wikstrom, Ekvall, \& Sandstrom, 1994).

Ao se analisar as diversas definições de criatividade encontradas ao longo do tempo, observa-se que, por vezes, são enfatizadas as habilidades cognitivas associadas à criatividade, por vezes os traços de personalidade ou, em outras ocasiões, o produto criativo (Nakano, 2003; S. M. Wechsler, 2008). Nesse sentido, a criatividade parece abranger certas capacidades mentais, entre elas a capacidade de mudar a maneira pela qual a pessoa aborda um problema, produzir ideias relevantes, ter uma visão além da situação imediata, redefinir problema (Runco, 2007). Nakano e Wechsler (2006) verificaram a queda da criatividade quando estudantes do ensino médio e superior foram comparados utilizando o teste Pensando Criativamente com Figuras. Também na pesquisa de padronização dos testes Pensando Criativamente com Figuras e Palavras houve influência da interação entre nível educacional e gênero, pois meninos obtiveram notas melhores no ensino médio, ao passo que o contrário aconteceu no ensino superior, no qual as meninas tiveram melhores resultados (S. M. Wechsler, 2004a, 2004b). Há estudos, entretanto, que verificaram um declínio na criatividade na velhice decorrente da redução da velocidade do processamento da informação, o que prejudicaria os idosos nos testes de criatividade (Ruth \& Birren, 1985).

Há estudos mostrando indícios de que inteligência e criatividade são habilidades que podem estar relacionadas. S. M. Wechsler, Nunes, Schelini, Ferreira e Pereira (2010) encontraram relação significativa entre a inteligência e a criatividade entre estudantes da primeira série do Ensino Fundamental à terceira série do Ensino Médio, tendo também observado que o desenvolvimento da inteligência ocorre de modo contínuo até 18 anos, o que não acontece com a criatividade. Entre os estudos com idosos pode-se 
Souza, A. A. F. \& Wechsler, S. M. (2013). Inteligência e Criatividade na Maturidade e Velhice.

citar o de Yassuda e Silva (2010), que encontraram relação entre o desempenho cognitivo, o humor e a satisfação com a vida para idosos participantes de programas de terceira idade. Já no trabalho de Oguzhanoglu e Osman (2005), foi realizada aplicação de psicodrama orientado de grupo em idosos que vivem enfermos em casa; como resultado observou-se um aumento de interesse, espontaneidade, criatividade. Conseqüentemente às características criativas houve melhora na comunicação, no relacionamento com os outros, na articulação, na emoção e no pensamento.

Tendo em vista a necessidade do estudo do desenvolvimento psicológico na fase da velhice, bem como do estudo da relação entre inteligência e criatividade na população idosa, este estudo teve por objetivo comparar as habilidades cognitivas e criativas entre pessoas na meia-idade (45 a 59 anos) e idosos (acima de 60 anos) e verificar a influência de sexo e nível de escolaridade nas medidas.

\section{Método}

\section{Participantes}

A amostra foi composta por 81 pessoas com idades variando de 45 a 83 anos, sendo 56 mulheres e 31 homens. O grupo de meia idade foi composto por 50 pessoas (16 homens e 34 mulheres), com idades variando de 45 a 59 anos (média=53,1 e desvio padrão 4,45). $O$ grupo de idosos foi composto por 31 pessoas ( 9 homens e 22 mulheres), idades variando de 60 a 83 anos (idade média $=63,4$ e desvio padrão 5,2 ) e $38,3 \%$ idosos. Quanto ao nível de escolaridade, entre as mulheres na meia-idade 55,9\% possuía Ensino Superior (Graduação ou Pós-Graduação), 29,4\% Ensino Médio e 14,7\% Ensino Fundamental. Por sua vez, $59,1 \%$ das participantes idosas tinham Ensino Fundamental, 31,8\% Ensino Médio e 9,1\% Ensino Superior. Entre os homens na faixa etária da meia-idade, 56,2\% possuíam o Ensino Superior, 37,5\% Ensino Médio e apenas uma pessoa tinha o Ensino Fundamental. Entre os participantes na velhice $77,8 \%$ tinham o Ensino Superior e $22,2 \%$ Ensino Fundamental.

A amostra foi composta por conveniência, tendo como critério de exclusão a presença de doenças degenerativas ou que afetem as funções cognitivas. Tal critério de exclusão foi verificado a partir de questionamento realizado com os participantes acerca do seu estado de saúde. Entrou-se em contato com dois grupos de terceira idade (um realizado pelo Serviço Social da Indústria - SESI, e um que frequentava o programa de uma Universidade Aberta a Terceira Idade - UNATI, na região do Alto Tietê/SP Cruzes), nos quais os participantes foram convidados a fazer parte do estudo.

\section{Instrumentos}

Para avaliação da inteligência foi utilizada a Bateria de Habilidades Intelectuais de Adultos (BAIAD) - baseada na Bateria de Habilidades Cognitivas Woodcock-Johnson, que teve adaptação para amostra brasileira de (S. M. Wechsler, Nunes, Schelini, Pasian, et al., 2010). Todos os testes verbais foram refeitos a partir da língua portuguesa falada no país bem como houve adaptação dos demais testes. A bateria possui 10 testes a partir dos quais são verificadas as habilidades cognitivas de acordo com a teoria CHC: Inteligência fluida (Gf), Inteligência cristalizada (Gc avaliada por 4 subtestes: vocabulário, sinônimos, antônimos e analogias), Memória de trabalho (Gsm), Memoria de recuperação a curto e longo prazo (Glr), Processamento visual (Gv), Velocidade de processamento (Gs).

A avaliação da criatividade foi feita por meio dos testes Pensando Criativamente com Figuras e Pensando Criativamente com Palavras (Torrance, 1990), que avaliam o potencial criativo por meio de dimensões cognitivas e afetivas expressas de modo figurativo e verbal (Torrance, 1966). Estes instrumentos foram validados para o país por S. M. Wechsler (2004a, 2004b), que demonstrou que as dimensões criativas avaliadas estavam relacionadas com a produção criativa reconhecida, não reconhecida e total entre brasileiros. O primeiro instrumento é composto por três atividades que avaliam a criatividade por meio de desenhos e o segundo de seis atividades escritas, as quais os participantes devem cumprir dentro de um tempo determinado. Esses instrumentos são capazes de avaliar indicadores cognitivos e emocionais entre as características criativas.

\section{Procedimentos}

\section{Coleta dos Dados}

O projeto foi aprovado pelo Comitê de Ética em Pesquisa com Seres Humanos da PUC-Campinas (Protocolo 0219/10). Os instrumentos foram aplicados individualmente em três sessões ou mais de acordo com a disponibilidade do participante. Foi feito um contato com os participantes para esclarecimentos e anuência na participação no estudo (através de assinatura do Termo de Consentimento Livre e Esclarecido). A coleta de dados, foi realizada nas dependências dos grupos de terceira idade (SESI, UNATI), em sala destinada especialmente para este fim. Na primeira sessão foi aplicada parte dos testes da BAIAD. A segunda sessão ocorreu de 5 a 7 dias após a primeira. Neste encontro foram aplicados os testes restantes da BAIAD. Na terceira sessão foram aplicados os testes Pensando Criativamente com Figuras e Pensando Criativamente com Palavras. Em alguns casos duas sessões foram insuficientes para a aplicação de toda a bateria BAIAD; tal ocorrência se deve ao fato de se tratar de uma bateria ainda em fase de padronização e, portanto, não há limite de tempo de realização dos testes. Por esse motivo alguns idosos demoravam muito tempo e duas sessões eram insuficientes para conclusão da bateria. Nesses casos foi marcado um encontro extra antes da terceira sessão.

\section{Análise dos Dados}

Após a coleta procedeu-se à correção dos testes e análise dos resultados. Os resultados foram trabalhados a partir dos escores brutos em inteligência e criatividade. 
Primeiramente foram analisadas as influências de sexo, faixa etária e nível educacional nos resultados por meio de Análise Multivariada e Univariada da Variância (MANOVA e ANOVA). A correlação de Pearson foi aplicada para verificar a relação entre inteligência e criatividade. Para os testes estatísticos utilizou-se o nível de significância de 0,05 .

\section{Resultados}

Tendo em vista que a BAIAD é composta por 11 sub-testes, optou-se por agrupar os resultados considerando: testes verbais (vocabulário, sinônimos, antônimos e analogias verbais), testes de memória (aprendizagem visual, números invertidos, memória auditiva para palavras e nú- meros e aprendizagem visual adiada) e outras habilidades (relações espaciais, formação de conceitos e combinação visual), os quais serão apresentados nos tópicos seguintes.

\section{Testes Verbais}

Para verificar os fatores que podiam influenciar o desempenho nos testes verbais foi realizada em um primeiro momento MANOVA dos quatro testes verbais com variáveis fixas sexo, escolaridade e faixa etária, o que revelou influência da escolaridade $(F=2,253, p \leq 0,027)$ e do sexo $(F=3,379, p \leq 0,014)$. A partir de tal resultado, foram realizadas Análises Univariadas da Variância para cada um dos testes verbais tendo também como variáveis fixas sexo, escolaridade e faixa etária, as quais estão apresentadas na Tabela 1.

Tabela 1

Análise da Variância nos Resultados de Inteligência e Criatividade

\begin{tabular}{lccccccc}
\hline Subtestes & Sexo & Escolaridade & $\begin{array}{c}\text { Faixa } \\
\text { Etária }\end{array}$ & $\begin{array}{c}\text { Sexo } X \\
\text { Escolaridade }\end{array}$ & $\begin{array}{c}\text { Sexo } X \\
\text { Faixa } \\
\text { Etária }\end{array}$ & $\begin{array}{c}\text { Escolaridade } X \\
\text { Faixa Etária }\end{array}$ & $\begin{array}{c}\text { Sexo } X \\
\text { Escolar } X \\
\text { Faixa Etária }\end{array}$ \\
\hline Vocabulário & 2,144 & $3,269^{*}$ & 0,172 & 0,038 & 0,486 & 1,798 & 0,122 \\
Sinônimos & 1,056 & $3,272^{*}$ & 0,212 & 0,699 & 0,900 & 0,582 & 0,375 \\
Antônimos & 0,214 & $7,141^{* *}$ & 0,384 & 0,899 & 0,437 & 0,577 & 0,039 \\
Analogias Verbais & 0,008 & $6,719^{* *}$ & 0,723 & 0,373 & 0,003 & 0,861 & 0,191 \\
Memória Auditiva Palavras & 0,363 & $6,591^{* *}$ & 0,189 & 2,408 & $6,720^{* *}$ & 2,480 & $5,430^{*}$ \\
Memória Auditiva Números & 0,006 & $3,812^{*}$ & 0,196 & 1,273 & 0,645 & 0,355 & $4,111^{*}$ \\
Aprendizagem Visual Adiada & 0,543 & 0,883 & 0,001 & 0,827 & $9,993^{* *}$ & $3,603^{*}$ & $3,954^{*}$ \\
Relações Espaciais & 0,200 & 1,633 & 2,246 & 0,301 & 0,028 & 0,219 & 2,201 \\
Formação de Conceitos & 0,370 & 1,554 & 0,228 & 0,536 & 0,032 & 1,044 & $4,599^{*}$ \\
Combinação Visual & 0,114 & $10,075^{* * *}$ & 1,717 & 0,027 & 0,198 & 0,138 & 0,100 \\
Índice Criativo Figural I & 0,315 & 2,239 & 0,795 & 0,107 & 2,275 & 0,759 & 0,761 \\
Índice Criativo Figural II & 0,677 & $3,247^{*}$ & 0,714 & 0,286 & 1,408 & 0,514 & 0,973 \\
Índice Criativo Verbal I & 0,986 & $3,380^{*}$ & 0,288 & 1,005 & 1,635 & 0,667 & 0,003 \\
Índice Criativo Verbal II & 0,986 & $3,467^{*}$ & 0,265 & 1,194 & 1,647 & 0,869 & 0,004 \\
\hline
\end{tabular}

${ }^{*} p \leq 0,05 ; *^{*} p \leq 0,01 ; * * * \mathrm{p} \leq 0,001$.

Os resultados mostraram que apenas o nível de escolaridade influencia significantemente os resultados verbais (Vocabulário, $F=3,269, p \leq 0,044$, Sinônimos, $F=3,272, p \leq 0,044$, Antônimos, $F=7,141, p \leq 0,002$ e Analogias Verbais, $F=6,719, p \leq 0,002)$. Nos testes citados, observou-se um aumento da pontuação conforme aumento da escolaridade, ou seja, quanto maior o nível educacional, maior o desempenho nos testes verbais. As médias ajustadas de cada sub-teste aplicado encontram-se na Tabela 2.

\section{Testes de Memória}

A BAIAD possui quatro testes que avaliam 3 tipos de memória: Trabalho (Testes Números Invertidos, Memória Auditiva para palavras e números), Recuperação de Curto Prazo (Aprendizagem Visual) e Longo Prazo (Aprendizagem Visual Adiada). A MANOVA dos testes de memória que compõem a BAIAD foi realizada tendo por fatores fixo sexo, faixa etária e nível de escolaridade. Os resultados demostraram que as interações sexo e faixa etária $(F=2,708$, $p \leq 0,031)$ e faixa etária e nível de escolaridade $(F=1,941$, 


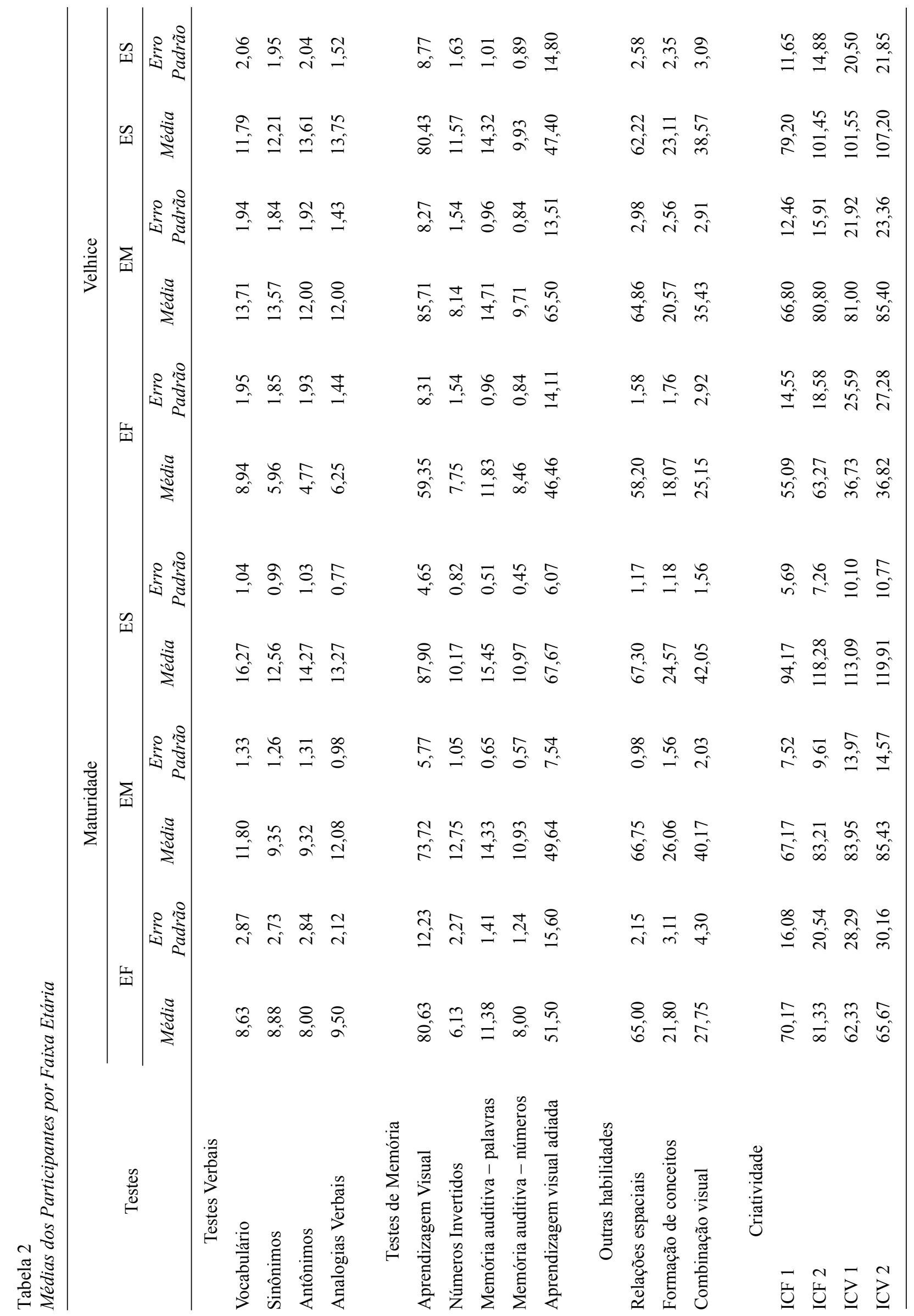


$p \leq 0,048)$ foram significativas. A partir desses resultados foram realizadas ANOVAS para cada teste de memória da BAIAD tendo por variáveis fixas sexo, escolaridade e faixa etária.

Observou-se que para os testes Números invertidos $(F=3,267, p \leq 0,044)$ e Memória Auditiva (palavras $F=6,591, p \leq 0,002$ e números $F=3,812, p \leq 0,027$ ) a escolaridade é fator significante que influencia os resultados. Além disto a interação entre escolaridade e faixa etária influenciou o teste de Números invertidos $(F=3,069$, $p \leq 0,053$ ) sendo que para o grupo na meia-idade houve um aumento na média do Ensino Fundamental para o Ensino Médio e um decréscimo no Ensino Superior (Tabela 2).

A avaliação da Memória Auditiva com Palavras teve também influência da interação entre sexo e faixa etária $(F=6,720, p \leq 0,01)$ e da interação entre escolaridade, sexo e faixa etária $(F=5,430, p \leq 0,05)$. Para os participantes na maturidade houve aumento de acordo com a escolaridade (fundamental=11,37, Médio=14,33, Superior=15,45) e para os idosos há aumento na pontuação entre Ensino Fundamental (média ajustada $=11,83$, erro padrão $=0,96$ ) e Médio (média ajustada $=14,71$, erro padrão $=0,95$ ) e decréscimo para Ensino Superior (média ajustada=14,32, erro padrão=1,01). Na Memória Auditiva com Números houve efeito da interação entre sexo, escolaridade e faixa etária $(F=4,11, p \leq 0,05)$. Os resultados quanto a memória auditiva números indicam, na amostra total, aumento de acordo com nível de escolaridade (Fundamental=8,23, erro padrão $=0,74$, Médio $=10,52$, erro padrão $=0,47, \mathrm{Su}-$ perior $=10,45$, erro padrão $=0,49$ ). Tais resultados indicam a necessidade de estudos para verificação do desenvolvimento da memória a partir da meia-idade considerando a interação desses três fatores.

Os resultados do teste Aprendizagem Visual Adiada, que avalia memória de recuperação a longo prazo, foram influenciados significantemente pelas interações entre sexo e faixa etária $(F=9,993, p \leq 0,003)$, escolaridade e faixa etária $(F=3,603, p \leq 0,034)$ e pela tríplice relação entre sexo, escolaridade e faixa etária $(F=3,954, p \leq 0,052)$. O grupo feminino na meia-idade apresentou queda na pontuação entre Ensino Fundamental (média ajustada $=90,00$, erro padrão $=15,60$ ) e Médio (média ajustada $=57,89$, erro padrão $=9,00$ ), com aumento no Ensino Superior (média ajustada $=63,76$, erro padrão $=6,55$ ). Entre as mulheres idosas há uma aumento na pontuação entre Ensino Fundamental (média $=16,909$, erro padrão=8,15) e Médio (média ajustada $=65,50$, erro padrão $=13,51)$ e queda para Ensino Superior (média ajustada $=28,00$, erro padrão $=27,02$ ). Para os homens na meia-idade houve aumento na pontuação de acordo com nível educacional e para os idosos houve queda entre Ensino Fundamental (média ajustada=16,00, erro padrão $=27,02$ ) e Superior (média ajustada $=66,80$, erro padrão=12,08).

\section{Outras Habilidades}

Os testes Relações Espaciais, Formação de Conceitos e Combinação Visual completam a bateria BAIAD.
Relações Espaciais avalia a habilidade de processamento visual, Formação de Conceitos avalia inteligência fluida e Combinação Visual avalia velocidade de processamento. A Análise Multivariada da Variância destes testes tendo como variáveis fixas sexo, faixa etária e escolaridade mostrou influência significante da escolaridade $(F=3,253$, $p \leq 0,005)$.

Foi realizada a ANOVA univariada para verificar em cada um dos testes os efeitos das escolaridade, sexo e nível de escolaridade, exceto para o teste Relações Espaciais. No teste Formação de conceitos a interação sexo vs escolaridade $v$ faixa etária foi significativa $(F=4,599, p \leq 0,036)$. Para Combinação visual a escolaridade é fator que influencia significantemente os resultados $(F=10,075, p \leq 0,001)$.

Considerando as médias ajustadas no teste formação de conceitos (Tabela 2) entre os participantes na maturidade houve um aumento na média entre Ensino Fundamental (média ajustada $=20,37$, erro padrão=3,61) e Ensino Médio (média ajustada $=25,81$, erro padrão $=1,67$ ), com decréscimo no Ensino Superior (média ajustada $=24,83$, erro padrão=1,31). Já entre os idosos houve uma queda na média entre Ensino Fundamental (média ajustada $=20,79$, erro padrão $=2,45$ ) e Ensino Médio (média ajustada $=20,57$, erro padrão $=2,44$ ) e aumentou para os participantes com Ensino Superior ( média ajustada $=25,57$, erro padrão $=2,59$ ). Para o teste combinação visual houve aumento na pontuação de acordo com aumento no nível de escolaridade, independente de faixa etária.

\section{Criatividade}

A análise da criatividade verbal é feita a partir de dois índices: Índice Criativo Verbal 1 (ICV 1), composto por Fluência, Flexibilidade, Originalidade e Elaboração e Índice Criativo Verbal 2 (ICV 2) que inclui além das características do ICV 1 Expressão de Emoção, Fantasia, Perspectiva Incomum e Uso de analogias e metáforas. Da mesma forma, a criatividade figural apresenta o Índice Criativo Figural 1 (ICF 1), composto por Fluência, Flexibilidade, Originalidade e Elaboração, e Índice Criativo Figural 2 (ICF 2), que inclui além das características citadas no ICF 1 Expressão de Emoção, Fantasia, Movimento, a Perspectiva Incomum, Perspectiva Interna, Uso de contexto, Combinações de Ideias, Extensão de limite e Títulos Expressivos.

Análise Multivariada da Variância dos índices de criatividade tendo como fatores fixos sexo, faixa etária e escolaridade não mostrou influência significante de nenhum desses fatores. Foram realizadas então Análises Univariadas da Variância tendo por fatores fixos sexo, faixa etária e escolaridade. Os resultados demostraram o efeito apenas da escolaridade no desempenho em criatividade (ICV 1, $F=3,380, p \leq 0,041$, ICV 2, $F=3,467$, $p \leq 0,038$, ICF $2, F=3,247, p \leq 0,046$ ), exceto no ICF 1 . Independente da faixa etária houve aumento no índice criativo de acordo com a escolaridade, sendo que os participantes com Ensino Superior obtiveram resultados mais altos (Tabela 2). 
Souza, A. A. F. \& Wechsler, S. M. (2013). Inteligência e Criatividade na Maturidade e Velhice.

\section{Relação entre Inteligência e Criatividade}

Os resultados do escore total na BAIAD foram correlacionados aos Índices de Criatividade (ICF 1, ICF 2, ICV 1 e ICV 2). Para verificar se havia diferenças na criatividade de acordo com o nível de desempenho na inteligência realizou-se as correlações com a amostra total e também com o dois grupos selecionados a partir da mediana do resultado total na BAIAD (285). Assim, a Tabela 3 apresenta as correlações de Pearson da amostra total BAIAD com os índices de criatividade bem como dos participantes com resultado na BAID superior e inferior à mediana.

Tabela 3

Correlação entre o Total da Bateria de Habilidades Intelectuais de Adultos e os Índices Criativos

\begin{tabular}{|c|c|c|c|c|c|}
\hline & & Ind. Cria & Ind. Cria & Ind. Cria. & Ind. Cria. \\
\hline & Total BAIAD & Figural I & Figural II & Verbal I & Verbal II \\
\hline Amostra Total BAIAD & $(n=77)$ & & & & \\
\hline Total BAIAD & ----- & $0,536^{* *}$ & $0,558^{* *}$ & $0,569^{* *}$ & $0,561^{* *}$ \\
\hline Índ Criativo Figural I & & ----- & $0,988^{* *}$ & $0,600^{* *}$ & $0,607^{* *}$ \\
\hline Ind. Criativ. Figural II & & & ----- & $0,657^{* *}$ & $0,662 * *$ \\
\hline Ind. Criativo Verbal I & & & & ---- & $0,999 * *$ \\
\hline Ind. Criativo Verbal II & & & & & ---- \\
\hline Abaixo mediana BAIAD & $(n=37)$ & & & & \\
\hline Total BAIAD & ------ & 0,309 & 0,311 & 0,342 & 0,343 \\
\hline Índ Criativo Figural I & & ------- & $0,994 * *$ & 0,332 & 0,346 \\
\hline Ind. Criativ. Figural II & & & ----- & $0,370^{*}$ & $0,383^{*}$ \\
\hline Ind. Criativo Verbal I & & & & ------ & $0,999 * *$ \\
\hline Ind. Criativo Verbal II & & & & & ---- \\
\hline Acima mediana BAIAD & $(n=40)$ & & & & \\
\hline Total BAIAD & ------- & $0,480^{* *}$ & $0,458^{* *}$ & 0,250 & 0,224 \\
\hline Índ Criativo Figural I & & ------- & $0,982 * *$ & $0,626^{* *}$ & $0,629 * *$ \\
\hline Ind. Criativ. Figural II & & & ------ & $0,675^{* *}$ & $0,677^{* *}$ \\
\hline Ind. Criativo Verbal I & & & & ----- & $0,998^{* *}$ \\
\hline Ind. Criativo Verbal II & & & & & ------ \\
\hline
\end{tabular}

$* p \leq 0,05 ; * * \leq \leq 0,01$.

Pode-se observar nos resultados da Tabela 3, considerando a amostra total, que todos os índices de criatividade estão correlacionados à inteligência, em um nível de significância de 0,01 , ou seja, para a amostra total quanto maior o resultado em inteligência maiores os índices de criatividade. Considerando a amostra que tem resultado de inteligência (BAIAD) acima da mediana, a inteligência apresenta-se correlacionada apenas aos índices de criatividade figurais, não havendo correlação com os índices de criatividade verbais. Já ao verificar o resultado em inteligência da amostra abaixo da mediana constata-se que a inteligência não está correlacionada a nenhum dos índices de criatividade. Assim sendo, a criatividade apresenta-se relacionada com patamares mais altos da inteligência.

Partindo do princípio que a criatividade verbal poderia estar relacionada ao desempenho dos testes verbais que avaliam a inteligência cristalizada, foi realizada a correlação entre o resultado nos testes verbais da BAIAD e os Índices Criativos Verbais. 
Tabela 4

Correlação entre o Total da Verbal da Bateria de Habilidades Intelectuais de Adultos e os Índices Criativos Verbais e entre Relações Espaciais e os Índices Criativos Figurais

\begin{tabular}{|c|c|c|c|c|c|c|}
\hline & Total Verbal & ICV 1 & ICV 2 & $\begin{array}{l}\text { Relações } \\
\text { Espaciais }\end{array}$ & ICF 1 & ICF 2 \\
\hline Total Verbal BAIAD & ----- & $0,583 *$ & $0,577^{*}$ & ----- & ----- & ----- \\
\hline Ind. Criativo Verbal I & & ---- & $0,999 *$ & ----- & ----- & ----- \\
\hline Ind. Criativo Verbal II & & & ----- & ----- & ----- & ----- \\
\hline Relações Espaciais & & & & ----- & $0,440^{*}$ & $0,450^{*}$ \\
\hline Índice Criativo Figural I & & & & & ----- & $0,988^{*}$ \\
\hline Índice Criativo Figural II & & & & & & ----- \\
\hline
\end{tabular}

Nota. Observação: amostra total.

${ }^{*} p \leq 0,01$.

Verificou-se na Tabela 4 que os Índices de Criatividade Verbais estão fortemente correlacionados ao total verbal da bateria de inteligência, o que corrobora a relação entre o conhecimento verbal e a expressão criativa verbal. Da mesma forma ficou evidente a relação entre os resultados no teste Relações Espaciais e os Índices Criativos Figurais 1 e 2. Portanto conforme aumento na habilidade espacial há aumento também no resultado em criatividade figural.

\section{Discussão}

Este estudo visou verificar o percurso das habilidades intelectuais e criativas em indivíduos nas fases da maturidade e velhice. Neste sentido comparou os efeitos das variáveis de gênero, escolaridade, faixa etária e suas interações no desempenho de adultos e idosos. Os resultados obtidos na avaliação da inteligência demonstraram a influência altamente significativa da variável nível educacional nos indivíduos estudados. Tais resultados corroboram aqueles encontrados por S. M. Wechsler, Nunes, Schelini, Ferreira, et al. (2010) nos quais foi também observada a influência da escolarização no desempenho intelectual de crianças e jovens. Assim sendo, o nível educacional, representado pela inteligência verbal e cristalizada principalmente, deve ser considerado por meio de normas específicas para diferentes faixas etárias nos testes que têm por finalidade a avaliação da inteligência.

Deve ser ressaltado que a influência do nível de escolarização ocorreu não somente para habilidades intelectuais mas também nos índices de criatividade figurativo e verbal. Esse aspecto mostra a relevância do desenvolvimento educacional não apenas na velhice mas desde a infância. Segundo Johnson et al. (2010), a capacidade mental tende a ser relativamente estável ao longo da vida refletindo aspectos como educação, classe social e circunstâncias ambientais. Portanto, pode ser que a função cognitiva desenvolvida na juventude contribua para o desempenho na velhice. A própria escolarização realizada desde a infância até a fase adulta faz com o se amplie o conhecimento básico para a busca independente de conhecimento, bem como leva à intelectualização e a diversificação das atividades desenvolvidas, por exemplo, para o lazer nas diferentes faixas etárias. Tais atividades podem favorecer a manutenção das funções cognitivas e criativas na meia-idade e velhice.

Os resultados aqui encontrados também são condizentes com os de Ribeiro et al. (2010) e Yassuda e Silva (2010). Portanto, a escolaridade já vem sendo relatada na literatura como um fator que influencia o desempenho intelectual de idosos em diferentes áreas. Entretanto, a contribuição do presente estudo está na comparação entre meia-idade e velhice, já que não se encontrou diferenças significativas de desempenho dos participantes de acordo com a idade. Tal fato indica que a escolaridade influencia mais a inteligência, de um modo geral, do que a faixa etária, contrariando estudos que enfatizam apenas o decréscimo do desempenho na velhice comparado à fase adulta.

Conclui-se que o nível de escolaridade é um fator determinante no desempenho nas habilidades cognitivas, sendo esse um fator de atenção no atendimento ao idoso, mostrando a importância não apenas social das Universidades Abertas à Terceira Idade. Promover espaços que, além da convivência, proporcionem aumento do conhecimento e manutenção das faculdades intelectuais pode favorecer os idosos tanto intelectualmente quanto do ponto de vista do bem estar psicológico. Da mesma forma faz-se importante o desenvolvimento educacional desde a infância como forma de prevenção cognitiva na velhice, já que o nível educacional alcançado na juventude influencia o desempenho em testes de habilidades cognitivas entre idosos (Plassman et al., 1995).

Alguns autores (Angelini et al., 1999; Flynn, 1998; Kaufman \& Kaufman, 1983) encontraram evidência de que a inteligência fluida aumenta de uma geração para outra, o que pode explicar o resultado superior dos idosos 
no teste Formação de Conceitos, que avalia esta habilidade, em relação às pessoas na meia-idade. Outros autores, entretanto, consideram a possibilidade de diminuição desse fator (Cunningham \& Clayton, 1973; Tranter \& Koutstaal, 2008), o que mostra essa ser uma área que ainda carente de estudos. Considerando-se as teorias de inteligência utilizadas para avaliações psicométricas, pode-se dizer que a inteligência fluida parece declinar após a idade de 21 anos, devido à degeneração das estruturas fisiológicas (Brody, 2000; Sattler, 2001). Porém esse aspecto não foi evidenciado no presente estudo em que a faixa etária isoladamente não influenciou o resultado na inteligência fluida.

Buscou-se verificar também o desempenho de pessoas na meia-idade e velhice em criatividade por meio dos índices criativos figurais e verbais. A escolaridade também demonstrou efeito altamente significativo em três dos quatro índices estudados. Considerando que foram encontradas associações significativas entre criatividade verbal e inteligência cristalizada e criatividade figural e inteligência viso-espacial com criatividade figural, pode-se compreender o impacto da escolaridade nestas medidas, já que também afetaram as demais em inteligência. Tal fato vem demonstrar a forte relação entre a criatividade e o percurso educacional, pois quanto maior o nível educacional, melhores resultados tem sido obtidos nos testes de criatividade, como tem sido observado nos estudos de Nakano e Wechsler (2006) e de S. M. Wechsler (2004a, 2004b).

Os questionamentos sobre as possíveis relações entre inteligência e criatividade existem há vários anos (Guilford \& Christensen, 1973; Puccio \& Murdock, 1999), visando analisar se estes conceitos seriam independentes ou construtos sobrepostos, havendo, portanto, um limiar no qual poderia ser esperada uma alta relação entre ambos. Neste estudo foi demonstrado que a criatividade (verbal e figural) e a inteligência estão relacionadas considerando todos os participantes deste estudo. Porém, quando se compara a amostra que está acima da mediana em inteligência, esta relação com a inteligência só ocorre com a criatividade figural. Assim sendo, o limiar do nível intelectual parece afetar mais a expressão da criatividade figurativa do que a expressão criativa verbal. Ainda que existam vários estudos que confirmam as associações entre inteligência e criatividade (Batey \& Furnham, 2009; Birren, 2009; Gregory, Nettelbeck, \& Wilson, 2010; Ruth \& Birren, 1985; Staudinger, Maciel, Smith, \& Baltes, 1998; Zimprich, Allemand, \& Dellenbach, 2009), as conclusões sobre este tema são ainda inconsistentes, como aponta Runco (2007).

Quanto à criatividade verbal é interessante notar que o grupo na meia-idade obteve médias sempre superiores ao grupo de idosos em criatividade verbal, o que não aconteceu na inteligência verbal em que alguns grupos de idosos tiveram resultados superiores aos da meia-idade. Vale lembrar que, embora se perceba essa diferença nas médias entre meia-idade e velhice não houve influência significante da faixa etária no desempenho verbal de inteligência nem de criatividade. Na pesquisa de padro- nização dos testes Pensando Criativamente com Palavras e Pensando Criativamente com Figuras também não foi encontrada diferença de acordo com idade, embora a pesquisa tenha sido realizada com adultos jovens (S. M. Wechsler, 2004a, 2004b). No estudo já citado de Ribeiro et al. (2010) também foi observado que a fluência verbal esteve fortemente correlacionada à escolaridade.

Há um fator que interfere diretamente o desenvolvimento da tarefa criativa que é o tempo marcado para a realização do teste. Na velhice, a rapidez e a destreza tendem a diminuir o que pode ter prejudicado o desempenho no teste de criatividade, que tem tempo marcado para execução da tarefa. Ruth e Birren (1985) observaram declínio da criatividade com a idade, o que foi atribuído à redução da velocidade do processamento da informação. No estudo foi observada a relação entre criatividade figural e rapidez de processamento, o que indica que tal fato pode influenciar os resultados nos testes, principalmente quanto se avaliam pessoas com idade avançada.

Deve-se considerar o fato de que este estudo teve como instrumento a bateria de inteligência de adultos, ainda em fase de validação. Assim sendo, várias limitações foram encontradas referentes à própria estrutura da bateria, como por exemplo, a sua extensão. O fato de se utilizar uma bateria de avaliação de inteligência composta por 10 testes que ainda não foram validados e por esse motivo se aplicava todos os itens tornou a tarefa de difícil execução, principalmente para os idosos. Além disso não se sabe se o instrumento para adultos está adequado para a população idosa. Esse aspecto indica a necessidade de estudos para que se identifique as condições ideais para a elaboração de instrumentos para idosos, considerando as limitações de visão, audição, motora, por exemplo. Um outro aspecto a ser considerado diz respeito à formatação ou diagramação dos testes de inteligência utilizados. Considerando que o teste foi criado para adultos não foram previstas possíveis limitações da velhice, tais como dificuldade motora na escrita (que foi um dos aspectos que exigiu aplicação individual para anotação das respostas por parte do aplicador), dificuldade de visão (alguns idosos responderam ao teste com auxílio de lupa), o que pode ter interferido, por exemplo, no teste de vocabulário em que figuras eram apresentadas para serem nomeadas.

Sugere-se a realização de estudos para a elaboração de uma bateria reduzida, com letras maiores, e que comparasse a administração com e sem tempo estipulado para identificar se essas características proporcionariam uma avaliação mais adequada à faixa etária idosa. Talvez a medida de criatividade utilizada com os idosos não tenha sido a mais adequada, nesse sentido sugere-se que em futuros estudos sejam utilizadas outras formas de medida da criatividade de acordo com os interesses de pessoas idosas. Entretanto, considerando que este grupo é bastante diverso, ainda faltam estudos a fim de identificar qual seria a forma mais adequada de avaliação da criatividade entre idosos.

Tendo em vista a influência da interação entre sexo, faixa etária e escolaridade no desempenho em memória, 
sugere-se que mais pesquisas sejam realizadas nesta área ampliando a análise do nível de escolaridade no desempenho intelectual e criativo. Por outro lado, a interação observada entre a inteligência e criatividade fornece orientações valiosas para a prática do profissional de psicologia. A promoção do bem-estar do idoso depende da manutenção do desempenho cognitivo, que deve ser cuidado desde a juventude, já que os idosos com maior nível de escolaridade tiveram resultados superiores aos demais. Recomenda-se também a realização de futuros estudos verificando a capacidade cognitiva a partir de uma amplitude maior de idade, a fim de se traçar o perfil do desenvolvimento cognitivo desde a fase adolescente até a velhice.

\section{Referências}

Aadlandsvik, R. (2007). Education, poetry, and the process of growing old. Educational Gerontology, 33(8), 665-678.

Alencar, E. S. M., \& Fleith, D. S. (2004). Inventário de práticas docentes que favorecem a criatividade no ensino superior. Psicologia e Reflexão, 17(1), 105-110.

Angelini, A. L., Alves, I. C. B., Custódio, E. M., Duarte, W. F., \& Duarte, J. L. M. (1999). Matrizes Progressivas Coloridas de Raven: Escala Especial. Manual. São Paulo, SP: Centro Editor de Testes e Pesquisas em Psicologia.

Batey, M. A., \& Furnham, A. (2009). The relationship between creativity, schizotypy and intelligence. Individual Differences Research, 7(4), 272-284.

Birren, J. E. (2009). Gifts and talents of elderly people: The persimmon's promise. In F. D. Horowitz, R. F. Subotnik, \& D. J. Matthews, The development of giftedness and talent across the life span. Washington, DC: American Psychological Association.

Brody, N. (2000). History of theories and measurements of intelligence. In R. J. Sternberg (Ed.), Handbook of intelligence (pp. 16-33). New York: Cambridge University Press.

Carroll, J. B. (1993). Human cognitive abilities: A survey of factor-analytic studies. New York: Cambridge University Press.

Cattell, R. B. (1941). Some theoretical issues in adult intelligence testing. Psychological Bulletin, 31, 161-179.

Cattell, R. B. (1971). Abilities: Their structure, growth and action. Boston, MA: Houghton Mifflin.

Colom, R., \& Flores-Mendonza, C. (2001). Inteligência y Memoria de Trabajo: la relación entre Fator G, Complejidad Cognitiva y Capacidad de Procesamiento. Psicologia: Teoria e Pesquisa, 17(1), 37-47.

Cunningham, W. R., \& Clayton, V. (1973). "Fluid" \& “crystallized" intelligence in the elderly. Proceedings of the Annual Convention of the American Psychological Association, 775776.

Flynn, J. R. (1984). The mean IQ of Americans: Massive gains 1932-1978. Psychological Bulletin, 95, 29-51.

Flynn, J. R. (1998). Raising IQ scores: Implications for the elderly. Australian Journal on Ageing, 17, 106-107.

Flynn, J. F. (2007). What is intelligence? New York: Cambridge University Press.

Foster, M. T. (1992). Experiencing a "creative high". Journal of Creative Behavior, 26(1), 29-39.

Godoy, M. de F. G. (1996). Criatividade e integração vital com idosos (Dissertação de mestrado, Pontifícia Universidade Católica de Campinas, SP, Brasil).
Gori, G., Pientini, S., \& Vespa, A. (2001). The selection of meaningful activities as a treatment for day-care in dementia. Archives of Gerontology and Geriatrics Supplement, 7, 207-212.

Gregory, T., Nettelbeck, T., \& Wilson, C. (2010). Openness to experience, intelligence, and successful ageing. Personality and Individual Differences, 48(8), 895-899.

Guilford, J. P., \& Christensen, P. R. (1973). The one-way relation between creative potential and IQ. Journal of Creative Behavior, 7, 247-252.

Horn, J. H. (1991). Measurement of intellectual capabilities: A review of theory. In K. S. McGrew, J. K. Werder, \& R. W. Woodcock (Eds.), WJ-R Technical Manual. Allen, TX: DLM.

Johnson, W., Gow, A. J., Corley, J., Starr, J. M., \& Deary, I. J. (2010). Location in cognitive and residential space at age 70 reflects a lifelong trait over parental and environmental circumstances: The Lothian birth cohort 1936. Intelligence, 38(4), 402-411.

Kaufman, A. S. (2009). IQ testing: 101 series. New York: Springer.

Kaufman, A. S., \& Kaufman, N. L. (1983). K-ABC interpretative manual. Circle Pines, MN: American Guidance Service.

McGrew, K. S., \& Flanagan, D. P. (1998). The inteligence test desk reference (ITDR): Gf-Gc cross-batery assessment. Needham Heights, MA: Allyn \& Bacon.

Muñoz-Sandoval, A., \& Woodcock, R. W. (2005). Bateria Woodcock-Muñoz III: proebas de habilidad cognitiva/ Bateria Woodcock-Muñoz III: proebas de aprovechamento. In S. M. Wechsler \& R. S. L. Guzzo (Eds.), Avaliação Psicológica: Perspectiva internacional (2. ed.). São Paulo, SP: Casa do Psicólogo.

Nakano, T. C. (2003). Teste Brasileiro de Criatividade Figural: Proposta de instrumento (Dissertação de mestrado, Pontifícia Universidade Católica de Campinas, SP, Brasil).

Nakano, T. de C., \& Wechsler, S. M. (2006). O percurso da criatividade figural do ensino médio ao ensino superior. Boletim de Psicologia, 56(125), 205-219.

Oguzhanoglu, N. K., \& Osman, O. (2005). Elderly, Nursing Homes and Life Voyages: A Psychodrama Group Study. Turk Psikiyatri Dergisi, 16(2), 124-132.

Oliveira-Castro, J. M., \& Oliveira-Castro, K. M. (2001). A função adverbial de "inteligência": Definições e usos em Psicologia. Psicologia: Teoria e Pesquisa, 17(3), 257-264.

Plassman, B. L., Welsh, K. A., Helms, M., Brandt, J., Page, W. F., \& Breitner, J. C. S. (1995). Intelligence and education as predictors of cognitive state in late life: A 50-year follow-up. Neurology, 45(8), 1446-1450.

Primi, R. (2002). Avanços na concepção psicométrica da inteligência. In F. C. Capovilla (Ed.), Neuropsicologia e aprendizagem: Uma abordagem multidisciplinar. São Paulo, SP: Sociedade Brasileira de Neuropsicologia.

Puccio, G. J., \& Murdock, M. C. (1999). Creativity assessment: Readings and resources. Buffalo, NY: Creative Education Foundation

Ribeiro, P. C. C., Oliveira, B. H. D., Cupertino, A. P. F. B., Néri, A. L., \& Yassuda, M. S. (2010). Desempenho de Idosos na Bateria Cognitiva CERAD: Relações com Variáveis Sociodemográficas e Saúde Percebida. Psicologia: Reflexão e Crítica, 23(1), 102-109.

Reisenwitz, T., Iyer, R., Kuhlmeier, D. B., \& Eastman, J. K. (2007). The elderly's Internet usage: An updated look. Journal of Consumer Marketing, 24(7), 406-418.

Runco, M. A. (2007). Creativity: Theories and themes, research, development and practice. Burlington, MA: Elsevier Academic Press. 
Ruth, J. E., \& Birren, J. E. (1985). Creativity in adulthood and old age: Relations to intelligence, sex and mode of testing. International Journal of Behavioral Development, 8(1), 99-109.

Sattler, J. M. (2001). Assessment of children-Cognitive application ( $4^{\text {th }}$ ed.). San Diego, CA: Jerome M. Sattler.

Spearman, C. (1927). The abilities of man: Their nature and measurement. New York: Macmillan.

Staudinger, U. M., Maciel, A. G., Smith, J., \& Baltes, P. B. (1998). What predicts wisdom-related performance? A first look at personality, intelligence, and facilitative experiential contexts. European Journal of Personality, 12(1), 1-17.

Thurnstone, L. L. (1938). Primary mental abilities. Chicago, IL: University Chicago Press.

Torrance, E. P. (1966). Torrance tests of creative thinking. Lexington, MA: Personnel Press.

Torrance, E. P. (1990). Torrance tests of creative thinking. Benseville, IL: Scholastic Testing Service.

Tranter, L. J., \& Koutstaal, W. (2008). Age and flexible thinking: An experimental demonstration of the beneficial effects of increased cognitively stimulating activity on fluid intelligence in healthy older adults. Aging, Neuropsychology, and Cognition, 15(2), 184-207.

Wechsler, D. (2003). WISC-IV. Wechsler intelligence scale for children ( $3^{\text {rd }}$ ed.). San Antonio. TX: Psychological Corporation. (Original work published 1949)

Wechsler, S. M. (2004a). Avaliação da criatividade por palavras. Campinas, SP: Pontifícia Universidade Católica de Campinas.

Wechsler, S. M. (2004b). A Avaliação da Criatividade por Figuras. Campinas, SP: Pontifícia Universidade Católica de Campinas.

Wechsler, S. M. (2008). Criatividade. Descobrindo e Encorajando (3. ed.). Campinas, SP: Pontifícia Universidade Católica de Campinas.

Wechsler, S. M., Nunes, C. S., Schelini, P. W., Pasian, S. R., Homsi, S. V., Moretti, L., \& Anache, A. A. (2010). Brazilian adaptation of the Woodcock-Johnson III: Cognitive Tests. School Psychology International, 31(4), 409-421.

Wechsler, S. M., Nunes, M. F. O., Schelini, P. W., Ferreira, A. A., \& Pereira, D. A. P. (2010). Criatividade e inteligência: Analisando semelhanças e discrepâncias no desenvolvimento. Estudo de Psicologia (Natal), 15(3), 243-250.

Wikstrom, B. M., Ekvall, G., \& Sandstrom, S. (1994). Stimulating the creativity of elderly institutionalized women through works of art. Creativity-Research-Journal, 7(2), 171-182.

Woodcock, R.W., McGrew, K. S., \& Mather, N. (2001). Woodcock-Johnson III. Itasca, IL: Riverside.

Yassuda, M. S., \& Silva, H. S. de (2010). Participação em programas para a terceira idade: Impacto sobre a cognição, humor e satisfação com a vida. Estudos de Psicologia (Campinas), 27(2), 207-214.

Zimprich, D., Allemand, M., \& Dellenbach, M. (2009). Openness to experience, fluid intelligence, and crystallized intelligence in middle-aged and old adults. Journal of Research in Personality, 43(3), 444-454. 